\title{
How to Limit the Beam-Induced Background in a Muon Collider
}

Danel Ally, Tova Holmes

\section{Abstract}

In order to continue our search for new particles, we need to increase the energy scales in our current colliders. It would be more efficient if we collided fundamental particles such as muons. With a muon collider, we could probe beyond the Standard Model physics (BSM) and also reveal new Higgs boson couplings. It would not suffer the same drastic radiation loss as a circular electron-positron collider, and all the energy would go toward collisions as opposed to a proton-proton collider. However, the beam-induced background (BIB) caused by muon decays would impact any signatures of new physics. Thus, we need to find ways to limit it. I will show you, if we make certain cuts on time and angle of travel on hits in the innermost layer of the tracker, we can decrease the BIB by $92.7 \%$ while only cutting $1.67 \%$ of the signal data.

\section{Introduction}

In order to probe new physics, we must go to higher energies; however, for proton-proton scattering, there is a limit. By colliding fundamental particles, we can maximize the energy of our collisions. Muon collisions with center-of-mass (CM) energy $14 \mathrm{TeV}$ would give us the same results as a proton-proton collision with CM energy of $100 \mathrm{TeV}$. As a result, a muon collider could probe BSM physics and search for new Higgs couplings, and because the mass of a muon is approximately 200 times that of an electron, a muon collider would not suffer the same drastic radiation loss as a circular electron-positron collider.

Despite the potential a muon collider might have in exploring hard to reach sectors of particle physics, there is one big drawback. The beam-induced background (BIB), which is caused by muon decays interacting with the detector overshadows any physics we would see from the muon collisions. For scale, one event produces approximately 500,000 BIB particles and approximately 100 hard scatter particles. Thus, it is of the utmost importance to reduce the amount of BIB that reaches the detector.

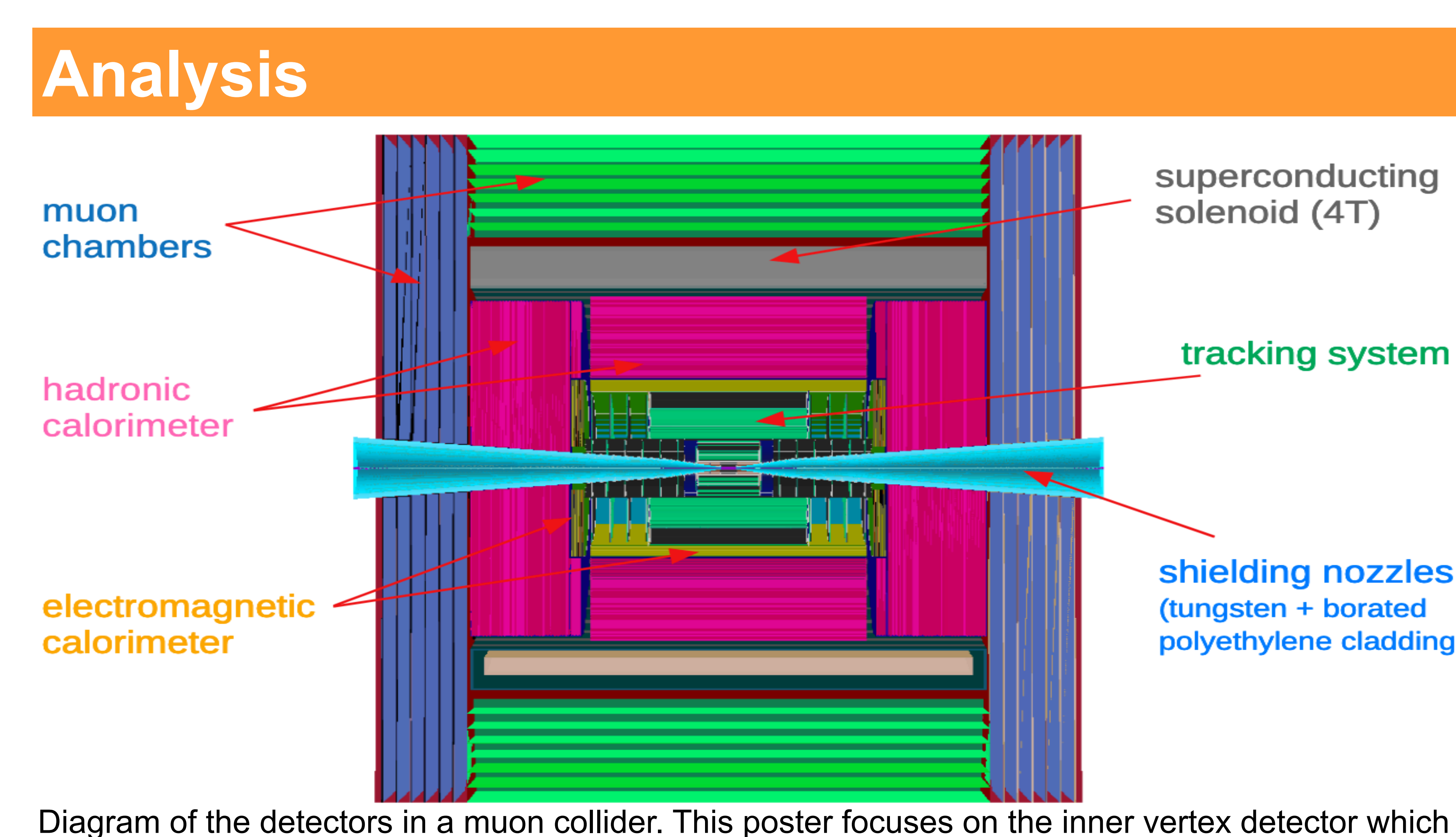

Diagram of the detectors in a muon collider. This poster
has 4 double-sensor barrel layers and is $130 \mathrm{~cm}$ long.

Because we want to look at the efficiency of the signal as we cut the BIB, we make a fiducial cut on the hard scatter data. We assume that any particles with momentum less than 1 $\mathrm{GeV}$ come from radiative effects and second-order particle interactions and cut them from our dataset.

In looking at different distributions of particles in the detector, we want to see if the signal agrees with the thought that it originated at the collision point. If we correct time for the time of flight (ToF), the time it takes a particle to reach the sensors assuming it originates at the collision point, We see that the signal data is close to 0 . If we make a cut on this value, |Time - ToF|, less than 20 ps, we can cut $90.7 \%$ of the BIB and only $1.67 \%$ of the signal.
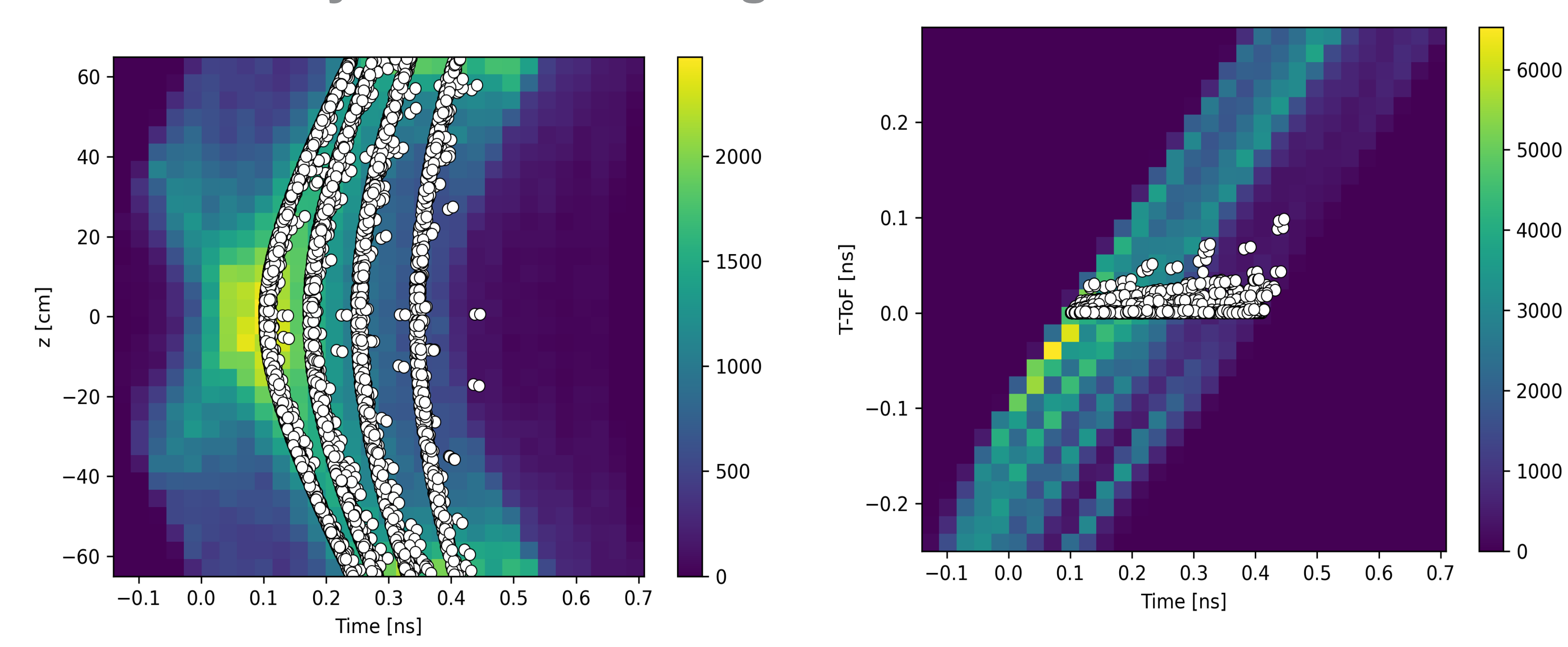

Histograms showing the position vs time at which the detector registers a hit cut (left) and the ToF correction (right)
minimal loss.
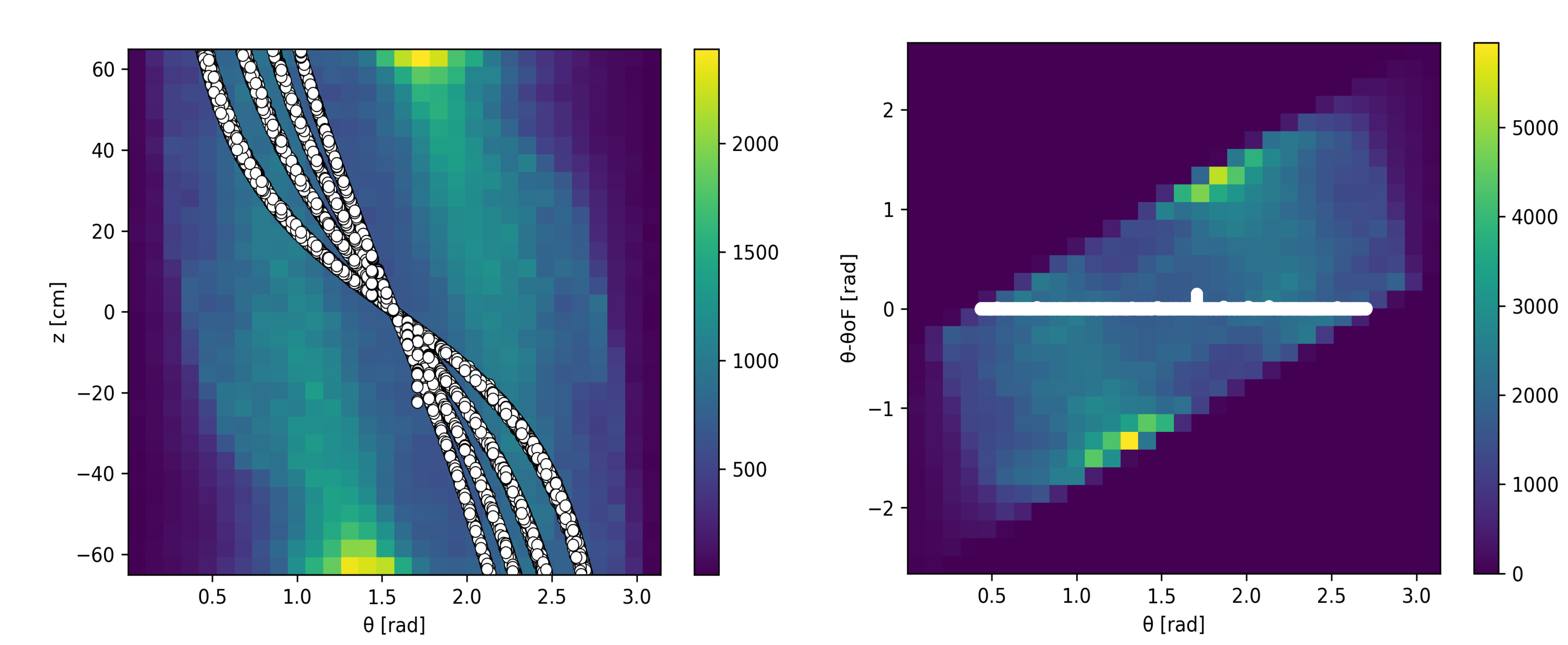

Histograms showing the angle of travel (left) with the expected angle correction (right). The correction shows that if we can attain the resolution, we can cut much of the BIB.

When we look at other values associated with the signal hits, we notice the same thing happening with a theta correction. $\theta$ in this context is what we're calling the angle of flight. If we correct it with the expected theta, $\theta_{\text {ex }}$, the angle of flight of the particles assuming they're coming from the collision point, we see again that the signal data is at 0 .

We are not certain of the angular resolution of our detector, so we cut on $\left|\theta-\theta_{\text {ex }}\right|$ less than one radian. Along with the time cut, the amount of BIB cut increases to $92.7 \%$ while no more of the signal is lost.

\section{Results}

\begin{tabular}{|c|c|c|c|c|c|c|}
\hline & \multicolumn{2}{|c|}{$1 \mathrm{Tad}$} & \multicolumn{2}{c|}{$0.1 \mathrm{rad}$} & \multicolumn{2}{c|}{$0.01 \mathrm{rad}$} \\
\hline $\mathrm{T}-\mathrm{ToF}\left\|<-\theta_{e x}\right\|<$ & $92.7 \%$ & $1.67 \%$ & $99.2 \%$ & $1.75 \%$ & $99.9 \%$ & $2.01 \%$ \\
\hline $20 \mathrm{ps}$ & $89.2 \%$ & $0.745 \%$ & $98.7 \%$ & $0.822 \%$ & $99.9 \%$ & $1.08 \%$ \\
\hline $30 \mathrm{ps}$ & $82.7 \%$ & $0.198 \%$ & $97.9 \%$ & $0.274 \%$ & $99.8 \%$ & $0.533 \%$ \\
\hline $50 \mathrm{ps}$ & $69.0 \%$ & $0 \%$ & $96.0 \%$ & $0.076 \%$ & $99.6 \%$ & $0.335 \%$ \\
\hline $100 \mathrm{ps}$ & $48.8 \%$ & $0 \%$ & $93.6 \%$ & $0.076 \%$ & $99.3 \%$ & $0.335 \%$ \\
\hline $200 \mathrm{ps}$ & & & \\
\hline Table showing how much of the BIB (left) we cut versus how much of the hard scatter (right) we
\end{tabular}

Table showing how much of the $\mathrm{BI}$
cut depending on different values. 